\title{
STABLE UNDER SPECIALIZATION SETS AND COFINITENESS
}

\author{
KAMRAN DIVAANI-AAZAR, HOSSEIN FARIDIAN AND MASSOUD TOUSI
}

\begin{abstract}
Let $R$ be a commutative noetherian ring, and $\mathcal{Z}$ a stable under specialization subset of $\operatorname{Spec}(R)$. We introduce a notion of $\mathcal{Z}$-cofiniteness and study its main properties. In the case $\operatorname{dim}(\mathcal{Z}) \leq 1$, or $\operatorname{dim}(R) \leq 2$, or $R$ is semilocal with $\operatorname{cd}(\mathcal{Z}, R) \leq 1$, we show that the category of $\mathcal{Z}$-cofinite $R$-modules is abelian. Also, in each of these cases, we prove that the local cohomology module $H_{\mathcal{Z}}^{i}(X)$ is $\mathcal{Z}$-cofinite for every homologically left-bounded $R$-complex $X$ whose homology modules are finitely generated and every $i \in \mathbb{Z}$.
\end{abstract}

\section{Contents}

1. Introduction

2. Preliminaries

3. Proof of Theorem 1.5

4. Proof of Theorem 1.6

References

\section{Introduction}

Throughout this paper, $R$ denotes a commutative noetherian ring with identity and $\mathcal{M}(R)$ flags the category of $R$-modules.

In his algebraic geometry seminars of 1961-2, Grothendieck founded the theory of local cohomology and raised, along the way, a few questions on the finiteness properties of the local cohomology modules; see $\mathrm{Gr}$, Conjectures 1.1 and 1.2]. He specifically asked whether the $R$ modules $\operatorname{Hom}_{R}\left(R / \mathfrak{a}, H_{\mathfrak{a}}^{i}(M)\right)$ were finitely generated for every ideal $\mathfrak{a}$ of $R$ and every finitely generated $R$-module $M$, which had been answered affirmatively in the same seminar when $(R, \mathfrak{m})$ is local and $\mathfrak{a}=\mathfrak{m}$. In 1969, Hartshorne provided a counterexample in [Ha1, Section 3], to show that this question does not have an affirmative answer in general. For a given ideal $\mathfrak{a}$ of $R$, Hartshorne defined an $R$-module $M$ to be $\mathfrak{a}$-cofinite if $\operatorname{Supp}_{R}(M) \subseteq \mathrm{V}(\mathfrak{a})$ and $\operatorname{Ext}_{R}^{i}(R / \mathfrak{a}, M)$ is finitely generated for every $i \geq 0$, and accordingly posed the following questions:

Question 1.1. Are the local cohomology modules $H_{\mathfrak{a}}^{i}(M)$, a-cofinite for every finitely generated $R$-module $M$ and every $i \geq 0$ ?

Question 1.2. Is the category $\mathcal{M}(R, \mathfrak{a})_{\text {cof }}$ of $\mathfrak{a}$-cofinite $R$-modules an abelian subcategory of $\mathcal{M}(R)$ ?

2010 Mathematics Subject Classification. 13D45; 13D07; 13 D09.

Key words and phrases. Abelian category; cofinite module; cohomological dimension; derived category; local cohomology module; stable under specialization set.

The research of the first author is supported by a grant from IPM (No. 95130212). 
By providing the following counterexample, he showed that the answers to these questions are negative in general.

Example 1.3. Let $k$ be a field, $R=k[X, Y][[U, V]], \mathfrak{a}=(U, V), \mathfrak{p}=(X V+Y U)$, and $T=R / \mathfrak{p}$. Then $R$ is a regular domain of dimension 4 and $T$ is a non-regular domain. It is shown that $\operatorname{Hom}_{R}\left(R / \mathfrak{a}, H_{\mathfrak{a}}^{2}(T)\right)$ is not finitely generated, so in particular, $H_{\mathfrak{a}}^{2}(T)$ is not a-cofinite. This takes care of Question 1.1. Furthermore, there is an exact sequence

$$
0 \rightarrow H_{\mathfrak{a}}^{1}(T) \rightarrow H_{\mathfrak{a}}^{2}(R) \rightarrow H_{\mathfrak{a}}^{2}(R) \rightarrow H_{\mathfrak{a}}^{2}(T) \rightarrow 0 .
$$

The local cohomology module $H_{\mathfrak{a}}^{2}(R)$ turns out to be $\mathfrak{a}$-cofinite, whereas $H_{\mathfrak{a}}^{2}(T)$ is not $\mathfrak{a}$-cofinite, answering Question 1.2.

Hartshorne further established affirmative answers to these questions in the case where $\mathfrak{a}$ is a principal ideal generated by a nonzerodivisor and $R$ is an a-adically complete regular ring of finite Krull dimension, and also in the case where $\mathfrak{a}$ is a prime ideal with $\operatorname{dim}(R / \mathfrak{a})=1$ and $R$ is a complete regular local ring; see [Ha1, Propositions 6.1 and 7.6, and Corollaries 6.3 and 7.7].

In the following years, Hartshorne's results on Questions 1.1 and 1.2 were systematically extended and polished by commutative algebra practitioners in several stages to take the following full-fledged culminating form.

Theorem 1.4. Let $\mathfrak{a}$ be an ideal of $R$, and $M$ a finitely generated $R$-module. Suppose that either of the following conditions are satisfied:

(i) $\operatorname{cd}(\mathfrak{a}, R) \leq 1$, or

(ii) $\operatorname{dim}(R / \mathfrak{a}) \leq 1$, or

(iii) $\operatorname{dim}(R) \leq 2$,

Then $H_{\mathfrak{a}}^{i}(M)$ is $\mathfrak{a}$-cofinite for every $i \geq 0$, and $\mathcal{M}(R, \mathfrak{a})_{\text {cof }}$ is an abelian subcategory of $\mathcal{M}(R)$.

For (i), see [Me2, Corollary 3.14] and [DFT, Theorem 2.2 (ii)]. For (ii), see [Me1, Theorem 2.6 and Corollary 2.12], [BNS, Corollary 2.8], and [BN, Corollary 2.7]. Finally for (iii), see [Me2, Theorems 7.10 and 7.4].

For an a-cofinite $R$-module $M$, it is known that the set $\operatorname{Ass}_{R}(M)$ is finite, and all its Bass numbers and Betti numbers with respect to every prime ideal of $R$ are also finite. Probing such finiteness properties has been a high-profile problem in commutative algebra; see e.g. [HK], [HS] and $[\mathrm{Ly}$.

Several authors have strived to extend the results of Theorem 1.4 to generalized local cohomology modules. However, it is folklore that all the generalizations $H_{\varphi}^{i}(M), H_{\mathcal{Z}}^{i}(M), H_{\mathfrak{a}, \mathfrak{b}}^{i}(M)$, $H_{\mathfrak{a}}^{i}(M, N), H_{\varphi}^{i}(M, N)$, and $H_{\mathfrak{a}, \mathfrak{b}}^{i}(M, N)$ of the local cohomology module $H_{\mathfrak{a}}^{i}(M)$ of an $R$-module $M$, are special cases of the local cohomology module $H_{\mathcal{Z}}^{i}(X)$ of an $R$-complex $X$ with support in a stable under specialization subset $\mathcal{Z}$ of $\operatorname{Spec}(R)$; see Remark 4.8. Therefore, any established result on $H_{\mathcal{Z}}^{i}(X)$ encompasses all the previously known results on each of these local cohomology modules. In this direction, we aspire to define the general notion of $\mathcal{Z}$-cofiniteness and extend Theorem 1.4 to $H_{\mathcal{Z}}^{i}(X)$. We specifically obtain the following results; see Theorems 3.13 , and 4.7.

Theorem 1.5. Let $\mathcal{Z}$ be a stable under specialization subset of $\operatorname{Spec}(R)$ such that either $R$ is semilocal with $\operatorname{cd}(\mathcal{Z}, R) \leq 1$, or $\operatorname{dim}(\mathcal{Z}) \leq 1$, or $\operatorname{dim}(R) \leq 2$. Then $\mathcal{M}(R, \mathcal{Z})_{\text {cof }}$ is an abelian subcategory of $\mathcal{M}(R)$. 
Theorem 1.6. Let $\mathcal{Z}$ a stable under specialization subset of $\operatorname{Spec}(R)$, and $X$ a homologically left-bounded R-complex $X$ with finitely generated homology modules. Assume that either $R$ is semilocal with $\operatorname{cd}(\mathcal{Z}, R) \leq 1$, or $\operatorname{dim}(\mathcal{Z}) \leq 1$, or $\operatorname{dim}\left(\operatorname{Supp}_{R}(X)\right) \leq 2$. Then $H_{\mathcal{Z}}^{i}(X)$ is $\mathcal{Z}$ cofinite for every $i \in \mathbb{Z}$.

\section{Preliminaries}

In this section, we first present some background material on complexes which will be used in the rest of the work. For more information, refer to $\mathrm{AF}$, $\mathrm{CFH}$, $\mathrm{Ha} 2$, [i1, and $\mathrm{Sp}$. In what follows, $\mathcal{C}(R)$ denotes the category of $R$-complexes.

The derived category $\mathcal{D}(R)$ is defined as the localization of the homotopy category $\mathcal{K}(R)$ with respect to the multiplicative system of quasi-isomorphisms. Simply put, an object in $\mathcal{D}(R)$ is an $R$-complex $X$ displayed in the standard homological style

$$
X=\cdots \rightarrow X_{i+1} \stackrel{\partial_{i+1}^{X}}{\longrightarrow} X_{i} \stackrel{\partial_{i}^{X}}{\longrightarrow} X_{i-1} \rightarrow \cdots,
$$

and a morphism $\varphi: X \rightarrow Y$ in $\mathcal{D}(R)$ is given by the equivalence class of a pair $(f, g)$ of morphisms $X \stackrel{g}{\leftarrow} U \stackrel{f}{\rightarrow} Y$ in $\mathcal{C}(R)$ with $g$ a quasi-isomorphism, under the equivalence relation that identifies two such pairs $(f, g)$ and $\left(f^{\prime}, g^{\prime}\right)$, whenever there is a diagram in $\mathcal{C}(R)$ as follows which commutes up to homotopy:

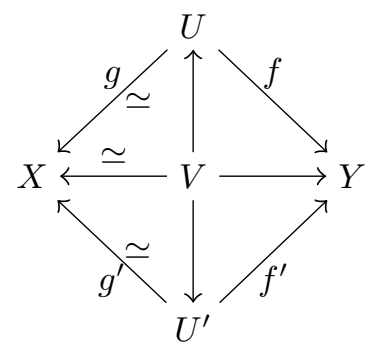

The isomorphisms in $\mathcal{D}(R)$ are marked by the symbol $\simeq$.

The derived category $\mathcal{D}(R)$ is triangulated. A distinguished triangle in $\mathcal{D}(R)$ is a triangle that is isomorphic to a triangle of the form

$$
X \stackrel{\mathfrak{L}(f)}{\longrightarrow} Y \stackrel{\mathfrak{L}(\varepsilon)}{\longrightarrow} \operatorname{Cone}(f) \stackrel{\mathfrak{L}(\varpi)}{\longrightarrow} \Sigma X,
$$

for some morphism $f: X \rightarrow Y$ in $\mathcal{C}(R)$ with the mapping cone sequence

$$
0 \rightarrow Y \stackrel{\varepsilon}{\rightarrow} \text { Cone }(f) \stackrel{\varpi}{\longrightarrow} \Sigma X \rightarrow 0,
$$

in which $\mathfrak{L}: \mathcal{C}(R) \rightarrow \mathcal{D}(R)$ is the canonical functor that is defined as $\mathfrak{L}(X)=X$ for every $R$-complex $X$, and $\mathfrak{L}(f)=\varphi$ where $\varphi$ is represented by the morphisms $X \stackrel{1^{X}}{\longleftarrow} X \stackrel{f}{\rightarrow} Y$ in $\mathcal{C}(R)$.

We let $\mathcal{D}_{\sqsubset}(R)$ (res. $\mathcal{D}_{\sqsupset}(R)$ ) denote the full subcategory of $\mathcal{D}(R)$ consisting of $R$-complexes $X$ with $H_{i}(X)=0$ for $i \gg 0$ (res. $i \ll 0$ ), and $D_{\square}(R):=\mathcal{D}_{\sqsubset}(R) \cap \mathcal{D}_{\sqsupset}(R)$. We further let $\mathcal{D}^{f}(R)$ denote the full subcategory of $\mathcal{D}(R)$ consisting of $R$-complexes $X$ with finitely generated homology modules. We also feel free to use any combination of the subscripts and the superscript as in $\mathcal{D}_{\square}^{f}(R)$, with the obvious meaning of the intersection of the two subcategories involved. Given an $R$-complex $X$, the standard notions

$$
\sup (X)=\sup \left\{i \in \mathbb{Z} \mid H_{i}(X) \neq 0\right\}
$$


and

$$
\inf (X)=\inf \left\{i \in \mathbb{Z} \mid H_{i}(X) \neq 0\right\}
$$

are frequently used, with the convention that $\sup (\emptyset)=-\infty$ and $\inf (\emptyset)=+\infty$.

An $R$-complex $P$ of projective modules is said to be semi-projective if the functor $\operatorname{Hom}_{R}(P,-)$ preserves quasi-isomorphisms. By a semi-projective resolution of an $R$-complex $X$, we mean a quasi-isomorphism $P \stackrel{\simeq}{\rightarrow} X$ in which $P$ is a semi-projective $R$-complex. Dually, an $R$-complex $I$ of injective modules is said to be semi-injective if the functor $\operatorname{Hom}_{R}(-, I)$ preserves quasiisomorphisms. By a semi-injective resolution of an $R$-complex $X$, we mean a quasi-isomorphism $X \stackrel{\simeq}{\rightarrow} I$ in which $I$ is a semi-injective $R$-complex. Semi-projective and semi-injective resolutions exist for any $R$-complex; see [CFH, Theorems 5.2.13 and 5.3.18]. Moreover, any right-bounded $R$-complex of projective modules is semi-projective, and any left-bounded $R$-complex of injective modules is semi-injective; see [CFH, Examples 5.2.7 and 5.3.11].

Let $X$ and $Y$ be two $R$-complexes. Then each of the functors $\operatorname{Hom}_{R}(X,-)$ and $\operatorname{Hom}_{R}(-, Y)$ on $\mathcal{C}(R)$ preserves homotopy equivalences, and thus enjoys a right total derived functor on $\mathcal{D}(R)$ $\left[\mathrm{CFH}\right.$, Theorem 7.1.14], together with a balance property, in the sense that $\mathbf{R} \operatorname{Hom}_{R}(X, Y)$ can be computed by

$$
\mathbf{R} \operatorname{Hom}_{R}(X, Y) \simeq \operatorname{Hom}_{R}(P, Y) \simeq \operatorname{Hom}_{R}(X, I),
$$

where $P \stackrel{\simeq}{\rightarrow} X$ is any semi-projective resolution of $X$, and $Y \stackrel{\simeq}{\rightarrow} I$ is any semi-injective resolution of $Y$. Moreover, we let $\operatorname{Ext}_{R}^{i}(X, Y):=H_{-i}\left(\mathbf{R} \operatorname{Hom}_{R}(X, Y)\right)$ for every $i \in \mathbb{Z}$.

Likewise, each of the functors $X \otimes_{R}-$ and $-\otimes_{R} Y$ on $\mathcal{C}(R)$ preserves homotopy equivalences, and thus enjoys a left total derived functor on $\mathcal{D}(R)$ [CFH, Theorem 7.2.20], together with a balance property, in the sense that $X \otimes_{R}^{\mathbf{L}} Y$ can be computed by

$$
X \otimes_{R}^{\mathbf{L}} Y \simeq P \otimes_{R} Y \simeq X \otimes_{R} Q,
$$

where $P \stackrel{\cong}{\rightarrow} X$ is any semi-projective resolution of $X$, and $Q \stackrel{\simeq}{\rightarrow} Y$ is any semi-projective resolution of $Y$. Moreover, we let $\operatorname{Tor}_{i}^{R}(X, Y):=H_{i}\left(X \otimes_{R}^{\mathbf{L}} Y\right)$ for every $i \in \mathbb{Z}$.

We next turn to the notion of a stable under specialization set. A subset $\mathcal{Z}$ of $\operatorname{Spec}(R)$ is said to be stable under specialization if $V(\mathfrak{p}) \subseteq \mathcal{Z}$ for every $\mathfrak{p} \in \mathcal{Z}$. For such a subset $\mathcal{Z}$, we set $F(\mathcal{Z}):=\{\mathfrak{a} \triangleleft R \mid V(\mathfrak{a}) \subseteq \mathcal{Z}\}$. If $M$ is an $R$-module, then clearly $\operatorname{Supp}_{R}(M)$ is a stable under specialization subset of $\operatorname{Spec}(R)$. Conversely, given any stable under specialization subset $\mathcal{Z}$ of $\operatorname{Spec}(R)$, one readily checks out that $\mathcal{Z}=\operatorname{Supp}_{R}\left(\bigoplus_{\mathfrak{a} \in F(\mathcal{Z})} R / \mathfrak{a}\right)$. In particular, $\mathrm{V}(\mathfrak{a})$ for an ideal $\mathfrak{a}$ of $R$, and any subset of $\operatorname{Max}(R)$ are stable under specialization subsets of $\operatorname{Spec}(R)$.

We finally recall the definition of the most general local cohomology functor. Given a stable under specialization subset $\mathcal{Z}$ of $\operatorname{Spec}(R)$, we let

$$
\Gamma_{\mathcal{Z}}(M):=\left\{x \in M \mid \operatorname{Supp}_{R}(R x) \subseteq \mathcal{Z}\right\}
$$

for an $R$-module $M$, and $\Gamma_{\mathcal{Z}}(f):=\left.f\right|_{\Gamma_{\mathcal{Z}}(M)}$ for an $R$-homomorphism $f: M \rightarrow N$. This provides us with the so-called $\mathcal{Z}$-torsion functor $\Gamma_{\mathcal{Z}}(-)$ on $\mathcal{M}(R)$, which extends, by termwise action, to a functor on $\mathcal{C}(R)$. The extended functor clearly preserves homotopy equivalences. Therefore, it enjoys a right total derived functor $\mathbf{R} \Gamma_{\mathcal{Z}}(-)$ on $\mathcal{D}(R)$ [CFH, Definition 6.6.12], that can be computed by $\mathbf{R} \Gamma_{\mathcal{Z}}(X) \simeq \Gamma_{\mathcal{Z}}(I)$, where $X \stackrel{\simeq}{\rightarrow} I$ is any semi-injective resolution of $X$. Besides, we define the $i$ th local cohomology module of $X$ with support in $\mathcal{Z}$ as $H_{\mathcal{Z}}^{i}(X):=H_{-i}\left(\mathbf{R} \Gamma_{\mathcal{Z}}(X)\right)$ for every $i \in \mathbb{Z}$. It is obvious that upon setting $\mathcal{Z}=\mathrm{V}(\mathfrak{a})$ for some ideal $\mathfrak{a}$ of $R$, we recover the usual local cohomology module with respect to $\mathfrak{a}$. 
It is straightforward to see that the set $F(\mathcal{Z})$ is a directed poset under reverse inclusion. Let $X$ be an $R$-complex and $X \stackrel{\simeq}{\rightarrow} I$ a semi-injective resolution of $X$. Then one can see by inspection that

$$
\Gamma_{\mathcal{Z}}\left(I_{i}\right)=\bigcup_{\mathfrak{a} \in F(\mathcal{Z})} \Gamma_{\mathfrak{a}}\left(I_{i}\right) \cong \underset{\mathfrak{a} \in F(\mathcal{Z})}{\lim _{\vec{a}}} \Gamma_{\mathfrak{a}}\left(I_{i}\right)
$$

for every $i \in \mathbb{Z}$, which in turn implies that $\Gamma_{\mathcal{Z}}(I) \cong \underset{\mathfrak{a} \in F(\mathcal{Z})}{\lim _{\mathfrak{a}}} \Gamma_{\mathfrak{a}}(I)$. Therefore, we have

$$
\begin{aligned}
& H_{\mathcal{Z}}^{i}(X) \cong H_{-i}\left(\mathbf{R} \Gamma_{\mathcal{Z}}(X)\right) \\
& \cong H_{-i}\left(\Gamma_{\mathcal{Z}}(I)\right) \\
& \cong H_{-i}\left({\underset{\mathfrak{l i m}}{\lim \in \mathcal{Z})}}_{\Gamma_{\mathfrak{a}}(I)}\right) \\
& \cong \lim _{\mathfrak{a} \in F(\mathcal{Z})} H_{-i}\left(\Gamma_{\mathfrak{a}}(I)\right) \\
& \cong \lim _{\mathfrak{a} \in F(\mathcal{Z})} H_{-i}\left(\mathbf{R} \Gamma_{\mathfrak{a}}(X)\right) \\
& \cong \underset{\mathfrak{a} \in F(\mathcal{Z})}{\lim _{\mathfrak{a}}} H_{\mathfrak{a}}^{i}(X)
\end{aligned}
$$

for every $i \in \mathbb{Z}$.

For a stable under specialization subset $\mathcal{Z}$ of $\operatorname{Spec}(R)$, we define the dimension of $\mathcal{Z}$ as

$$
\operatorname{dim}(\mathcal{Z}):=\sup \{\operatorname{dim}(R / \mathfrak{a}) \mid \mathfrak{a} \in F(\mathcal{Z})\} .
$$

Also, we define the cohomological dimension of an $R$-complex $X$ with respect to $\mathcal{Z}$ as

$$
\operatorname{cd}(\mathcal{Z}, X):=\sup \left\{i \in \mathbb{Z} \mid H_{\mathcal{Z}}^{i}(X) \neq 0\right\} .
$$

Now, we are ready to define the general notion of $\mathcal{Z}$-cofiniteness. Recall that the support of an $R$-complex $X$ is defined to be $\operatorname{Supp}_{R}(X)=\bigcup_{i \in \mathbb{Z}} \operatorname{Supp}_{R}\left(H_{i}(X)\right)$.

Definition 2.1. Let $\mathcal{Z}$ be a stable under specialization subset of $\operatorname{Spec}(R)$. An $R$-complex $X \in \mathcal{D}(R)$ is said to be $\mathcal{Z}$-cofinite if $\operatorname{Supp}_{R}(X) \subseteq \mathcal{Z}$ and $\mathbf{R} \operatorname{Hom}_{R}(R / \mathfrak{a}, X) \in \mathcal{D}^{f}(R)$ for every $\mathfrak{a} \in F(\mathcal{Z})$.

We denote the full subcategory of $\mathcal{M}(R)$ consisting of $\mathcal{Z}$-cofinite $R$-modules by $\mathcal{M}(R, \mathcal{Z})_{\text {cof }}$. The next result lays on some characterizations of $\mathcal{Z}$-cofinite complexes.

Lemma 2.2. Let $\mathcal{Z}$ be a stable under specialization subset of $\operatorname{Spec}(R)$, and $X \in \mathcal{D}(R)$ with $\operatorname{Supp}_{R}(X) \subseteq \mathcal{Z}$. Consider the following conditions:

(a) $X$ is $\mathcal{Z}$-cofinite.

(b) $\mathbf{R} \operatorname{Hom}_{R}(Y, X) \in \mathcal{D}^{f}(R)$ for every $Y \in \mathcal{D}_{\square}^{f}(R)$ with $\operatorname{Supp}_{R}(Y) \subseteq \mathcal{Z}$.

(c) $(R / \mathfrak{a}) \otimes_{R}^{\mathbf{L}} X \in \mathcal{D}^{f}(R)$ for every $\mathfrak{a} \in F(\mathcal{Z})$.

(d) $Y \otimes_{R}^{\mathbf{L}} X \in \mathcal{D}^{f}(R)$ for every $Y \in \mathcal{D}_{\square}^{f}(R)$ with $\operatorname{Supp}_{R}(Y) \subseteq \mathcal{Z}$.

Then the following assertions hold:

(i) If $X \in \mathcal{D}_{\sqsubset}(R)$, then (a) and (b) are equivalent.

(ii) If $X \in \mathcal{D}_{\sqsupset}(R)$, then (c) and (d) are equivalent.

(iii) If $X \in \mathcal{D}_{\square}(R)$, then all the assertions are equivalent. 
Proof. (i): Suppose that (a) holds and $Y \in \mathcal{D}_{\square}^{f}(R)$ with $\operatorname{Supp}_{R}(Y) \subseteq \mathcal{Z}$. Let $\mathfrak{a}=$ $\operatorname{ann}_{R}\left(\oplus_{i \in \mathbb{Z}} H_{i}(Y)\right)$. Then $V(\mathfrak{a})=\operatorname{Supp}_{R}(Y) \subseteq \mathcal{Z}$, so $\mathfrak{a} \in F(\mathcal{Z})$. Therefore, $\mathbf{R} \operatorname{Hom}_{R}(R / \mathfrak{a}, X) \in$ $\mathcal{D}^{f}(R)$. Now, WW, Proposition 7.2] implies that $\mathbf{R} \operatorname{Hom}_{R}(Y, X) \in \mathcal{D}^{f}(R)$. The converse is clear.

(ii): Similar to (i) using [WW, Proposition 7.1].

(iii): Fix $\mathfrak{a} \in F(\mathcal{Z})$. Then [WW, Proposition 7.4] yields that $\mathbf{R} \operatorname{Hom}_{R}(R / \mathfrak{a}, X) \in \mathcal{D}^{f}(R)$ if and only if $(R / \mathfrak{a}) \otimes_{R}^{\mathbf{L}} X \in \mathcal{D}^{f}(R)$.

We collect some basic properties of $\mathcal{Z}$-cofinite $R$-complexes in the following result. Its first part indicates that in the case where $\mathcal{Z}=V(\mathfrak{a})$, our definition of $\mathcal{Z}$-cofiniteness coincides with the usual notion of $\mathfrak{a}$-cofiniteness.

Lemma 2.3. Let $\mathcal{Z}$ be a stable under specialization subset of $\operatorname{Spec}(R)$ and $X \in \mathcal{D}_{\sqsubset}(R)$. Then the following assertions hold:

(i) If $\mathfrak{a}$ is an ideal of $R$, then $X$ is $V(\mathfrak{a})$-cofinite if and only if $\operatorname{Supp}_{R}(X) \subseteq V(\mathfrak{a})$ and $\mathbf{R} \operatorname{Hom}_{R}(R / \mathfrak{a}, X) \in \mathcal{D}^{f}(R)$.

(ii) If $H_{i}(X)$ is $\mathcal{Z}$-cofinite for every $i \in \mathbb{Z}$, then $X$ is $\mathcal{Z}$-cofinite.

(iii) If $X \in \mathcal{D}_{\sqsubset}^{f}(R)$, then $\mathbf{R} \Gamma_{\mathcal{Z}}(X)$ is $\mathcal{Z}$-cofinite.

(iv) If $X$ is $\mathcal{Z}$-cofinite, then the Bass number $\mu^{i}(\mathfrak{p}, X)$ is finite for every $\mathfrak{p} \in \operatorname{Spec}(R)$ and every $i \in \mathbb{Z}$.

(v) If $X \in \mathcal{D}_{\square}(R)$ and $X$ is $\mathcal{Z}$-cofinite, then the Betti number $\beta_{i}(\mathfrak{p}, X)$ is finite for every $\mathfrak{p} \in \operatorname{Spec}(R)$ and every $i \in \mathbb{Z}$.

Proof. (i): Suppose that $\operatorname{Supp}_{R}(X) \subseteq V(\mathfrak{a})$ and $\mathbf{R} \operatorname{Hom}_{R}(R / \mathfrak{a}, X) \in \mathcal{D}^{f}(R)$. Let $\mathfrak{b} \in F(V(\mathfrak{a}))$. It follows that $\operatorname{Supp}_{R}(R / \mathfrak{b}) \subseteq V(\mathfrak{a})$. Now, by [WW, Proposition 7.2] we are through. The converse is clear.

(ii): Since $H_{i}(X)$ is $\mathcal{Z}$-cofinite for every $i \in \mathbb{Z}$, we have

$$
\operatorname{Supp}_{R}(X)=\bigcup_{i \in \mathbb{Z}} \operatorname{Supp}_{R}\left(H_{i}(X)\right) \subseteq \mathcal{Z} .
$$

Let $\mathfrak{a} \in F(\mathcal{Z})$. The spectral sequence

$$
E_{p, q}^{2}=\operatorname{Ext}_{R}^{p}\left(R / \mathfrak{a}, H_{-q}(X)\right) \underset{p}{\Rightarrow} \operatorname{Ext}_{R}^{p+q}(R / \mathfrak{a}, X)
$$

from the proof of [Ha1, Proposition 6.2], together with the assumption that $E_{p, q}^{2}$ is finitely generated for every $p, q \in \mathbb{Z}$, conspire to imply that $\operatorname{Ext}_{R}^{p+q}(R / \mathfrak{a}, X)$ is finitely generated, i.e. $X$ is $\mathcal{Z}$-cofinite.

(iii): It is clear that $\operatorname{Supp}_{R}\left(\mathbf{R} \Gamma_{\mathcal{Z}}(X)\right) \subseteq \mathcal{Z}$. Since $X$ is homologically left-bounded, there exists a left-bounded semi-injective resolution $X \stackrel{\simeq}{\rightarrow} I$ of $X$ invoking [CFH, Theorem 5.3.26]. As $I$ is an $R$-complex of injective modules, and $\Gamma_{\mathcal{Z}}\left(I_{i}\right) \cong \underset{\mathfrak{a} \in F(\mathcal{Z})}{\lim _{\mathfrak{a}}} \Gamma_{\mathfrak{a}}\left(I_{i}\right)$ for every $i \in \mathbb{Z}$, we see that $\Gamma_{\mathcal{Z}}(I)$ is a left-bounded $R$-complex of injective modules, and thus $\Gamma_{\mathcal{Z}}(I)$ is semi-injective.

Fix $\mathfrak{a} \in F(\mathcal{Z})$. For every $R$-module $\operatorname{Hom}_{R}\left(R / \mathfrak{a}, \Gamma_{\mathcal{Z}}\left(I_{i}\right)\right)$ in the $R$-complex $\operatorname{Hom}_{R}\left(R / \mathfrak{a}, \Gamma_{\mathcal{Z}}(I)\right)$, we have

$$
\operatorname{Hom}_{R}\left(R / \mathfrak{a}, \Gamma_{\mathcal{Z}}\left(I_{i}\right)\right) \cong \operatorname{Hom}_{R}\left(R / \mathfrak{a}, \Gamma_{\mathfrak{a}}\left(I_{i}\right)\right) \cong \operatorname{Hom}_{R}\left(R / \mathfrak{a}, I_{i}\right) .
$$


Indeed, if $N$ is a submodule of $M$ containing $\Gamma_{\mathfrak{a}}(M)$, then every $R$-homomorphism $f: R / \mathfrak{a} \rightarrow N$ has its image in $\Gamma_{\mathfrak{a}}(M)$. Hence one has

$$
\begin{aligned}
\mathbf{R H o m}_{R}\left(R / \mathfrak{a}, \mathbf{R} \Gamma_{\mathcal{Z}}(X)\right) & \simeq \mathbf{R} \operatorname{Hom}_{R}\left(R / \mathfrak{a}, \Gamma_{\mathcal{Z}}(I)\right) \\
& \simeq \operatorname{Hom}_{R}\left(R / \mathfrak{a}, \Gamma_{\mathcal{Z}}(I)\right) \\
& \simeq \operatorname{Hom}_{R}(R / \mathfrak{a}, I) \\
& \simeq \mathbf{R} \operatorname{Hom}_{R}(R / \mathfrak{a}, X)
\end{aligned}
$$

But $X \in \mathcal{D}_{\sqsubset}^{f}(R)$, so $\mathbf{R} \operatorname{Hom}_{R}(R / \mathfrak{a}, X) \in \mathcal{D}_{\sqsubset}^{f}(R)$, and thus the assertion follows.

(iv): For every $i \in \mathbb{Z}$, we have

$$
\mu^{i}(\mathfrak{p}, X):=\operatorname{rank}_{R_{\mathfrak{p}} / \mathfrak{p} R_{\mathfrak{p}}}\left(\operatorname{Ext}_{R_{\mathfrak{p}}}^{i}\left(R_{\mathfrak{p}} / \mathfrak{p} R_{\mathfrak{p}}, X_{\mathfrak{p}}\right)\right) .
$$

If $\mathfrak{p} \notin \mathcal{Z}$, then $\mathfrak{p} \notin \operatorname{Supp}_{R}(X)$, so $\mu^{i}(\mathfrak{p}, X)=0$. If $\mathfrak{p} \in \mathcal{Z}$, then $\mathrm{V}(\mathfrak{p}) \subseteq \mathcal{Z}$, so by definition, $\mathbf{R} \operatorname{Hom}_{R}(R / \mathfrak{p}, X) \in \mathcal{D}^{f}(R)$, whence $\mu^{i}(\mathfrak{p}, X)<\infty$.

(v): For every $i \in \mathbb{Z}$, we have

$$
\beta_{i}(\mathfrak{p}, X):=\operatorname{rank}_{R_{\mathfrak{p}} / \mathfrak{p} R_{\mathfrak{p}}}\left(\operatorname{Tor}_{i}^{R_{\mathfrak{p}}}\left(R_{\mathfrak{p}} / \mathfrak{p} R_{\mathfrak{p}}, X_{\mathfrak{p}}\right)\right) .
$$

If $\mathfrak{p} \notin \mathcal{Z}$, then as in (iv), $\beta_{i}(\mathfrak{p}, X)=0$. If $\mathfrak{p} \in \mathcal{Z}$, then Lemma 2.2 (iii) implies that $(R / \mathfrak{p}) \otimes_{R}^{\mathbf{L}} X \in$ $\mathcal{D}^{f}(R)$, thereby $\beta_{i}(\mathfrak{p}, X)<\infty$.

\section{Proof of Theorem 1.5}

Our aim in this section is to prove Theorem 1.5, see Theorem 3.13. Corollary [3.5] is the main ingredient in the proof of the first part of Theorem 3.13. To prove it, we need Lemmas $3.1,3.2$ and 3.4 .

Lemma 3.1. Let $\mathcal{Z}$ be a stable under specialization subset of $\operatorname{Spec}(R)$. Let $M$ be an $R$-module such that $\operatorname{Ass}_{R}(M) \cap \mathcal{Z} \cap \operatorname{Max}(R)$ is a finite set. Then the following assertions are equivalent:

(i) $\left(0:_{M} \mathfrak{a}\right)$ is an artinian $R$-module for every $\mathfrak{a} \in F(\mathcal{Z})$.

(ii) $\Gamma_{\mathfrak{a}}(M)$ is an artinian $R$-module for every $\mathfrak{a} \in F(\mathcal{Z})$.

(iii) $\Gamma_{\mathcal{Z}}(M)$ is an artinian $R$-module.


is artinian, so using Melkersson's Criterion [Me3, Theorem 1.3], we conclude that $\Gamma_{\mathfrak{a}}(M)$ is artinian.

(ii) $\Rightarrow$ (iii): Set

$$
\mathfrak{J}:=\bigcap_{\mathfrak{m} \in \operatorname{Ass}_{R}(M) \cap \mathcal{Z} \cap \operatorname{Max}(R)} \mathfrak{m} .
$$

Since $\operatorname{Ass}_{R}(M) \cap \mathcal{Z} \cap \operatorname{Max}(R)$ is a finite set, we see that $V(\mathfrak{J})=\operatorname{Ass}_{R}(M) \cap \mathcal{Z} \cap \operatorname{Max}(R)$, so that $\mathfrak{J} \in F(\mathcal{Z})$. Therefore, $\Gamma_{\mathfrak{J}}(M)$ is an artinian $R$-module.

Now, let $\mathfrak{a} \in F(\mathcal{Z})$. Then $\Gamma_{\mathfrak{a}}(M)$ is an artinian $R$-module by the assumption. Let $x \in \Gamma_{\mathfrak{a}}(M)$. As $R x$ is artinian, it turns out that

$$
\begin{aligned}
\operatorname{Ass}_{R}(R x) & \subseteq \operatorname{Ass}_{R}(M) \cap V(\mathfrak{a}) \cap \operatorname{Max}(R) \\
& \subseteq \operatorname{Ass}_{R}(M) \cap \mathcal{Z} \cap \operatorname{Max}(R) \\
& =V(\mathfrak{J}) .
\end{aligned}
$$


Hence $x \in \Gamma_{\mathfrak{J}}(M)$. This yields that

$$
\Gamma_{\mathcal{Z}}(M)=\bigcup_{\mathfrak{a} \in F(\mathcal{Z})} \Gamma_{\mathfrak{a}}(M) \subseteq \Gamma_{\mathfrak{J}}(M) \subseteq \Gamma_{\mathcal{Z}}(M),
$$

thereby $\Gamma_{\mathcal{Z}}(M)=\Gamma_{\mathfrak{J}}(M)$ is artinian.

(iii) $\Rightarrow$ (i): Clear, since $\left(0:_{M} \mathfrak{a}\right) \subseteq \Gamma_{\mathcal{Z}}(M)$ for every $\mathfrak{a} \in F(\mathcal{Z})$.

Lemma 3.2. Let $\mathcal{Z}$ be a stable under specialization subset of $\operatorname{Spec}(R)$. Let $M$ be an $R$-module, and $r \geq 0$ an integer. Consider the following conditions:

(a) $H_{\mathcal{Z}}^{i}(M)$ is an artinian $R$-module for every $0 \leq i \leq r$.

(b) $\operatorname{Ext}_{R}^{i}(R / \mathfrak{a}, M)$ is an artinian $R$-module for every $\mathfrak{a} \in F(\mathcal{Z})$ and for every $0 \leq i \leq r$.

Then the following assertions hold:

(i) (a) implies (b).

(ii) If $\operatorname{Supp}_{R}(M) \cap \mathcal{Z} \cap \operatorname{Max}(R)$ is a finite set, then (a) and (b) are equivalent.

Proof. Let

$$
I: 0 \rightarrow I_{0} \stackrel{\partial_{0}^{I}}{\longrightarrow} I_{-1} \stackrel{\partial_{-1}^{I}}{\longrightarrow} I_{-2} \rightarrow \cdots
$$

be a minimal injective resolution of $M$. Given any $\mathfrak{a} \in F(\mathcal{Z})$, consider the two $R$-complexes

$$
\begin{gathered}
\operatorname{Hom}_{R}(R / \mathfrak{a}, I): 0 \rightarrow \operatorname{Hom}_{R}\left(R / \mathfrak{a}, I_{0}\right) \stackrel{\operatorname{Hom}_{R}\left(R / \mathfrak{a}, \partial_{0}^{I}\right)}{\longrightarrow} \operatorname{Hom}_{R}\left(R / \mathfrak{a}, I_{-1}\right) \\
\stackrel{\operatorname{Hom}_{R}\left(R / \mathfrak{a}, \partial_{-1}^{I}\right)}{\longrightarrow} \operatorname{Hom}_{R}\left(R / \mathfrak{a}, I_{-2}\right) \rightarrow \cdots,
\end{gathered}
$$

and

$$
\Gamma_{\mathcal{Z}}(I): 0 \rightarrow \Gamma_{\mathcal{Z}}\left(I_{0}\right) \stackrel{\Gamma_{\mathcal{Z}}\left(\partial_{0}^{I}\right)}{\longrightarrow} \Gamma_{\mathcal{Z}}\left(I_{-1}\right) \stackrel{\Gamma_{\mathcal{Z}}\left(\partial_{-1}^{I}\right)}{\longrightarrow} \Gamma_{\mathcal{Z}}\left(I_{-2}\right) \rightarrow \cdots
$$

One can easily check that $\operatorname{ker}\left(\operatorname{Hom}_{R}\left(R / \mathfrak{a}, \partial_{-i}^{I}\right)\right)$ is an essential submodule of $\operatorname{Hom}\left(R / \mathfrak{a}, I_{-i}\right)$, and $\operatorname{ker}\left(\Gamma_{\mathcal{Z}}\left(\partial_{-i}^{I}\right)\right)$ is an essential submodule of $\Gamma_{\mathcal{Z}}\left(I_{-i}\right)$ for every $i \geq 0$.

Let

$$
X: 0 \rightarrow X_{0} \stackrel{\partial_{0}^{X}}{\longrightarrow} X_{-1} \stackrel{\partial_{-1}^{X}}{\longrightarrow} X_{-2} \rightarrow \cdots
$$

be an $R$-complex such that $\operatorname{ker} \partial_{-i}^{X}$ is an essential submodule of $X_{-i}$ for every $i \geq 0$. For any given $r \geq 0$, [Me2, Lemma 5.4] yields that $X_{-i}$ is an artinian $R$-module for every $0 \leq i \leq r$ if and only if $H_{-i}(X)$ is an artinian $R$-module for every $0 \leq i \leq r$. In the remainder of the proof, we apply this twice.

Now, we prove the following:

(i): Let $\mathfrak{a} \in F(\mathcal{Z})$. Applying the discussion above to the $R$-complex $\Gamma_{\mathcal{Z}}(I)$, we see that $\Gamma_{\mathcal{Z}}\left(I_{-i}\right)$ is artinian for every $0 \leq i \leq r$. Since

$$
\operatorname{Hom}_{R}\left(R / \mathfrak{a}, I_{-i}\right) \cong\left(0:_{I_{-i}} \mathfrak{a}\right) \subseteq \Gamma_{\mathcal{Z}}\left(I_{-i}\right)
$$

it is obvious that $\operatorname{Hom}_{R}\left(R / \mathfrak{a}, I_{-i}\right)$ is artinian for every $0 \leq i \leq r$. This shows that $\operatorname{Ext}_{R}^{i}(R / \mathfrak{a}, M)$ is artinian for every $0 \leq i \leq r$.

(ii): Another application of the discussion above to the $R$-complex $\operatorname{Hom}_{R}(R / \mathfrak{a}, I)$, yields that $\operatorname{Hom}_{R}\left(R / \mathfrak{a}, I_{-i}\right)$ is artinian for every $\mathfrak{a} \in F(\mathcal{Z})$ and $0 \leq i \leq r$. Since $I$ is a minimal injective resolution of $M$, one can see that $\operatorname{Supp}_{R}\left(I_{-i}\right) \subseteq \operatorname{Supp}_{R}(M)$ for every $i \geq 0$. Therefore, we can use Lemma 3.1 to deduce that $\Gamma_{\mathcal{Z}}\left(I_{-i}\right)$ is artinian for every $0 \leq i \leq r$. A fortiori, $H_{\mathcal{Z}}^{i}(M)$ is artinian for every $0 \leq i \leq r$. 
Part (i) of the next example shows that the finiteness condition on the sets $\operatorname{Ass}_{R}(M) \cap \mathcal{Z} \cap$ $\operatorname{Max}(R)$ and $\operatorname{Supp}_{R}(M) \cap \mathcal{Z} \cap \operatorname{Max}(R)$ cannot be removed from Lemmas 3.1 and 3.2 respectively. Part (ii) of this example also demonstrates that unlike $\mathfrak{a}$-cofinite modules, a $\mathcal{Z}$-cofinite module can have infinitely many associated prime ideals.

Example 3.3. (i) Let $R$ be a ring with infinitely many maximal ideals. Let $\mathcal{Z}:=\operatorname{Max}(R)$, and $M:=\bigoplus_{\mathfrak{m} \in \mathcal{Z}} R / \mathfrak{m}$. Then $F(\mathcal{Z})=\{\mathfrak{a} \triangleleft R \mid \operatorname{dim}(R / \mathfrak{a}) \leq 0\}$. Thus for any given proper ideal $\mathfrak{a} \in F(\mathcal{Z})$, there are finitely many maximal ideals in $V(\mathfrak{a})$, say $\mathfrak{m}_{1}, \ldots, \mathfrak{m}_{n}$. It follows that $\Gamma_{\mathfrak{a}}(M)=\bigoplus_{i=1}^{n} R / \mathfrak{m}_{i}$. Now $\Gamma_{\mathfrak{a}}(M)$ is artinian, while $\Gamma_{\mathcal{Z}}(M)=M$ fails to be artinian as it contains infinitely many direct summands.

(ii) Let $R$ be a Gorenstein ring of finite dimension $d$ such that $\mathcal{Z}:=\{\mathfrak{m} \in \operatorname{Max}(R) \mid$ ht $(\mathfrak{m})=$ $d\}$ is an infinite set. Then by [HD, Remark 2.12], it turns out that

$$
H_{\mathcal{Z}}^{j}(R) \cong \begin{cases}\bigoplus_{\mathfrak{m} \in \mathcal{Z}} E_{R}(R / \mathfrak{m}) & \text { if } j=d \\ 0 & \text { if } j \neq d .\end{cases}
$$

Thus $\operatorname{Ext}_{R}^{i}\left(R / \mathfrak{a}, H_{\mathcal{Z}}^{j}(R)\right)$ is finitely generated for every $\mathfrak{a} \in F(\mathcal{Z})$ and every $i, j \geq 0$. It follows that $H_{\mathcal{Z}}^{j}(R)$ is $\mathcal{Z}$-cofinite for every $j \geq 0$, whereas $H_{\mathcal{Z}}^{d}(R)$ is not artinian and $\operatorname{Ass}_{R}\left(H_{\mathcal{Z}}^{d}(R)\right)$ is not finite.

Lemma 3.4. Let $R$ be a semilocal ring with Jacobson radical $\mathfrak{J}, \mathcal{Z}$ a stable under specialization subset of $\operatorname{Spec}(R)$, and $M$ an $R$-module. Let $(-)^{\vee}:=\operatorname{Hom}_{R}\left(-, E_{R}(R / \mathfrak{J})\right)$ be the Matlis duality functor. Then the following assertions are equivalent for any given $r \geq 0$ :

(i) $H_{\mathcal{Z}}^{i}\left(M^{\vee}\right)$ is an artinian $R$-module for every $0 \leq i \leq r$.

(ii) $\operatorname{Ext}_{R}^{i}\left(R / \mathfrak{a}, M^{\vee}\right)$ is an artinian $R$-module for every $\mathfrak{a} \in F(\mathcal{Z})$ and every $0 \leq i \leq r$.

(iii) $\operatorname{Tor}_{i}^{R}(R / \mathfrak{a}, M)$ is a finitely generated $R$-module for every $\mathfrak{a} \in F(\mathcal{Z})$ and every $0 \leq i \leq r$.

Proof. (i) $\Leftrightarrow$ (ii): Follows from Lemma 3.2

One can easily see that $E_{R}(R / \mathfrak{J}) \cong \bigoplus_{\mathfrak{m} \in \operatorname{Max}(R)} E_{R}(R / \mathfrak{m})$ is an artinian injective cogenerator for $R$. Fix $\mathfrak{a} \in F(\mathcal{Z})$ and $0 \leq i \leq r$, and let $N:=\operatorname{Tor}_{i}^{R}(R / \mathfrak{a}, M)$ for the rest of the proof. Then by [으, Corollary 10.63], we have $N^{\vee} \cong \operatorname{Ext}_{R}^{i}\left(R / \mathfrak{a}, M^{\vee}\right)$.

(ii) $\Rightarrow$ (iii): Let $\operatorname{Max}(R)=\left\{\mathfrak{m}_{1}, \ldots, \mathfrak{m}_{n}\right\}$ and set $T:=\widehat{R}^{\mathfrak{J}}$ and $T_{j}:=\widehat{R_{\mathfrak{m}_{j}}}$ for every $j=$ $1, \ldots, n$. We know that $T \cong \prod_{j=1}^{n} T_{j}$ and $T$ is a $\mathfrak{J} T$-adically complete semilocal ring with $\operatorname{Max}(T)=\left\{\mathfrak{m}_{j} T \mid j=1, \ldots, n\right\}$. Any $\mathfrak{J}$-torsion $R$-module possesses a $T$-module structure in such a way that a subset is an $R$-submodule if and only if it is a $T$-submodule. In particular, $N^{\vee}$ and $E_{R}(R / \mathfrak{J})$ are artinian $T$-modules. Moreover, one may easily check that the two $T$ modules $E_{R}(R / \mathfrak{J})$ and $E_{T}(T / \mathfrak{J} T)$ are isomorphic and $\mathfrak{J} T$ is the Jacobson radical of $T$. Putting everything together, we obtain:

$$
\begin{aligned}
N^{\vee} & \cong \operatorname{Hom}_{R}\left(N, E_{R}(R / \mathfrak{J})\right) \\
& \cong \operatorname{Hom}_{R}\left(N, E_{T}(T / \mathfrak{J} T)\right) \\
& \cong \operatorname{Hom}_{R}\left(N, \operatorname{Hom}_{T}\left(T, E_{T}(T / \mathfrak{J} T)\right)\right) \\
& \cong \operatorname{Hom}_{T}\left(N \otimes_{R} T, E_{T}(T / \mathfrak{J} T)\right) .
\end{aligned}
$$


Applying the Matlis Duality Theorem over the $\operatorname{ring} T$ [CW Proposition 4 (c)], we deduce that $N \otimes_{R} T$ is a finitely generated $T$-module and by the faithfully flatness of the completion map $\theta_{R}^{\mathfrak{J}}: R \rightarrow T$, we infer that $N$ is a finitely generated $R$-module.

(iii) $\Rightarrow$ (ii): There is an exact sequence

$$
R^{n} \rightarrow N \rightarrow 0
$$

which yields the exact sequence

$$
0 \rightarrow N^{\vee} \rightarrow E_{R}(R / \mathfrak{J})^{n}
$$

It follows that $\operatorname{Ext}_{R}^{i}\left(R / \mathfrak{a}, M^{\vee}\right) \cong N^{\vee}$ is an artinian $R$-module.

For any given $R$-module $N$, one may easily check that $\operatorname{cd}(\mathcal{Z}, N) \leq \operatorname{dim}(R)$.

Corollary 3.5. Let $R$ be a semilocal ring, $\mathcal{Z}$ a stable under specialization subset of $\operatorname{Spec}(R)$, and $M$ an $R$-module with $\operatorname{Supp}_{R}(M) \subseteq \mathcal{Z}$. Then the following assertions are equivalent:

(i) $M$ is $\mathcal{Z}$-cofinite.

(ii) $\operatorname{Tor}_{i}^{R}(R / \mathfrak{a}, M)$ is finitely generated for every $\mathfrak{a} \in F(\mathcal{Z})$ and every $i \geq 0$.

(iii) $H_{\mathcal{Z}}^{i}\left(M^{\vee}\right)$ is artinian for every $i \geq 0$.

(iv) $H_{\mathcal{Z}}^{i}\left(M^{\vee}\right)$ is artinian for every $0 \leq i \leq \operatorname{cd}\left(\mathcal{Z}, M^{\vee}\right)$.

(v) $\operatorname{Ext}_{R}^{i}\left(R / \mathfrak{a}, M^{\vee}\right)$ is artinian for every $\mathfrak{a} \in F(\mathcal{Z})$ and every $0 \leq i \leq \operatorname{cd}\left(\mathcal{Z}, M^{\vee}\right)$.

(vi) $\operatorname{Tor}_{i}^{R}(R / \mathfrak{a}, M)$ is finitely generated for every $\mathfrak{a} \in F(\mathcal{Z})$ and every $0 \leq i \leq \operatorname{cd}\left(\mathcal{Z}, M^{\vee}\right)$.

Proof. (i) $\Leftrightarrow$ (ii): Follows from Lemma 2.2 (iii).

(ii) $\Leftrightarrow$ (iii): Follows from Lemma 3.4 .

(iii) $\Leftrightarrow$ (iv): Obvious.

(iv) $\Leftrightarrow(\mathrm{v}) \Leftrightarrow(\mathrm{vi})$ : Follows from Lemma 3.4

The following two lemmas are our essential tools in the proof of the second part of Theorem 3.13

Lemma 3.6. Let $\mathcal{Z}$ be a stable under specialization subset of $\operatorname{Spec}(R)$, and $M$ an $R$-module with $\operatorname{Supp}_{R}(M) \subseteq \mathcal{Z}$ and $\operatorname{dim}_{R}(M) \leq 1$. Then the following assertions are equivalent:

(i) $M$ is $\mathcal{Z}$-cofnite.

(ii) $\operatorname{Hom}_{R}(R / \mathfrak{a}, M)$ and $\operatorname{Ext}_{R}^{1}(R / \mathfrak{a}, M)$ are finitely generated for every $\mathfrak{a} \in F(\mathcal{Z})$.

Proof. Follows from the equivalence (i) $\Leftrightarrow($ iii) in $[\mathrm{AB}$, Lemma 2.6].

In the sequel, we use the straightforward observation that if any two modules in a short exact sequence are $\mathcal{Z}$-cofinite, then so is the third.

Lemma 3.7. Let $\mathcal{Z}$ be a stable under specialization subset of $\operatorname{Spec}(R)$. Assume that either

(i) An $R$-module $M$ is $\mathcal{Z}$-cofinite whenever $\operatorname{Supp}_{R}(M) \subseteq \mathcal{Z}$ and $\operatorname{Ext}_{R}^{i}(R / \mathfrak{a}, M)$ is finitely generated for $i=0,1$ and every $\mathfrak{a} \in F(\mathcal{Z})$; or

(ii) An $R$-module $M$ is $\mathcal{Z}$-cofinite whenever $\operatorname{Supp}_{R}(M) \subseteq \mathcal{Z}$ and $\operatorname{Tor}_{i}^{R}(R / \mathfrak{a}, M)$ is finitely generated for $i=0,1$ and every $\mathfrak{a} \in F(\mathcal{Z})$,

holds. Then $\mathcal{M}(R, \mathcal{Z})_{\text {cof }}$ is an abelian subcategory of $\mathcal{M}(R)$. 
Proof. (i): Let $f: M \rightarrow N$ be an $R$-homomorphism between $\mathcal{Z}$-cofinite modules. We should prove that both $\operatorname{ker} f$ and coker $f$ are $\mathcal{Z}$-cofinite. In view of the short exact sequences

$$
0 \rightarrow \operatorname{im} f \rightarrow N \rightarrow \operatorname{coker} f \rightarrow 0,
$$

and

$$
0 \rightarrow \operatorname{ker} f \rightarrow M \rightarrow \operatorname{im} f \rightarrow 0,
$$

it suffices to show that $\operatorname{ker} f$ is $\mathcal{Z}$-cofinite. Let $\mathfrak{b} \in F(\mathcal{Z})$. From (3.7.1), we deduce that $\operatorname{Hom}_{R}(R / \mathfrak{b}, \operatorname{im} f)$ is finitely generated. Now, (3.7.2) yields the exact sequence

$$
\begin{gathered}
0 \rightarrow \operatorname{Hom}_{R}(R / \mathfrak{b}, \operatorname{ker} f) \rightarrow \operatorname{Hom}_{R}(R / \mathfrak{b}, M) \rightarrow \operatorname{Hom}_{R}(R / \mathfrak{b}, \operatorname{im} f) \rightarrow \\
\operatorname{Ext}_{R}^{1}(R / \mathfrak{b}, \operatorname{ker} f) \rightarrow \operatorname{Ext}_{R}^{1}(R / \mathfrak{b}, M) .
\end{gathered}
$$

Thus $\operatorname{Hom}_{R}(R / \mathfrak{b}, \operatorname{ker} f)$ and $\operatorname{Ext}_{R}^{1}(R / \mathfrak{b}, \operatorname{ker} f)$ are finitely generated. Next, our assumption in (i) implies that $\operatorname{ker} f$ is $\mathcal{Z}$-cofinite. Note that $\operatorname{Supp}_{R}(\operatorname{ker} f) \subseteq \mathcal{Z}$.

(ii): It is similar to the proof of (i), and so we leave it to the reader.

To prove the third part of Theorem 3.13, we need Lemmas 3.8, 3.9, 3.10, and 3.11,

Lemma 3.8. Let $S$ be a module-finite R-algebra. Let $\mathfrak{a}$ be an ideal of $R$, and $M$ an $S$-module. Then the $R$-module $\operatorname{Ext}_{R}^{i}(R / \mathfrak{a}, M)$ is finitely generated for every $i \geq 0$ if and only if the $S$-module $\operatorname{Ext}_{S}^{i}(S / \mathfrak{a} S, M)$ is finitely generated for every $i \geq 0$.

Proof. The proof of [DM, Proposition 2] establishes the claim. Note that the assumption on the supports is not used in that proof.

Let $\mathfrak{a}$ be an ideal of $R$. An $R$-module $M$ is said to be $\mathfrak{a}$-Ext-finite if $\operatorname{Ext}_{R}^{i}(R / \mathfrak{a}, M)$ is finitely generated for every $i \geq 0$.

Lemma 3.9. Let $\mathfrak{a}$ be a proper ideal of $R$, and $M$ an $R$-module. Suppose that $\operatorname{dim}(R) \leq 1$. Then the following assertions hold:

(i) If $\left(0:_{M} \mathfrak{a}\right)$ is finitely generated, then $M$ is $\mathfrak{a}$-Ext-finite.

(ii) The class of $\mathfrak{a}$-Ext-finite $R$-modules is closed under taking submodules, quotients, and extensions.

Proof. (i): There is an integer $n \geq 1$ such that $\Gamma_{\mathfrak{a}}(R)=\left(0::_{R} \mathfrak{a}^{n}\right)$. Then $\bar{M}:=M /\left(0:_{M} \mathfrak{a}^{n}\right)$ is a module over the ring $\bar{R}:=R / \Gamma_{\mathfrak{a}}(R)$. Let $\overline{\mathfrak{a}}$ be the image of $\mathfrak{a}$ in $\bar{R}$. Then $\overline{\mathfrak{a}}$ contains an $\bar{R}$-regular element and thus $\operatorname{dim}(\bar{R} / \overline{\mathfrak{a}})=0$. We note that as $\left(0:_{M} \mathfrak{a}\right)$ is finitely generated, one may check that $\left(0:_{M} \mathfrak{a}^{i}\right)$ is finitely generated for every $i \geq 1$. So, $\left(0:_{\bar{M}} \overline{\mathfrak{a}}\right)=\left(0:_{M} \mathfrak{a}^{n+1}\right) /\left(0:_{M} \mathfrak{a}^{n}\right)$ is a finitely generated $R$-module. It follows that $(0: \bar{M} \overline{\mathfrak{a}})$ is a finitely generated $\bar{R} / \overline{\mathfrak{a}}$-module. As $\bar{R} / \overline{\mathfrak{a}}$ is artinian, we see that $(0: \bar{M} \overline{\mathfrak{a}})$ is artinian as an $\bar{R} / \overline{\mathfrak{a}}$-module, and thus as an $\bar{R}$-module. By Me3, Theorem 1.3], it follows that $\Gamma_{\overline{\mathfrak{a}}}(\bar{M})$ is an artinian $\bar{R}$-module. $\operatorname{But} \operatorname{dim}_{\bar{R}}(\bar{M}) \leq 1$, so $\underline{\mathrm{Me} 2}$, Proposition 5.1] implies that $H_{\overline{\mathfrak{a}}}(\bar{M})$ is an artinian $\overline{\mathfrak{a}}$-cofinite $\bar{R}$-module. It now follows from Lemma 3.2 that $\operatorname{Ext}_{\bar{R}}^{i}(\bar{R} / \overline{\mathfrak{a}}, \bar{M})$ is an artinian $\bar{R}$-module for every $i \geq 0$. But $\bar{R} / \overline{\mathfrak{a}}$ is artinian, so $\operatorname{Ext}_{\bar{R}}^{i}(\bar{R} / \overline{\mathfrak{a}}, \bar{M})$ has finite length for every $i \geq 0$. It follows from Lemma 3.8 that $\operatorname{Ext}_{R}^{i}(R / \mathfrak{a}, \bar{M})$ is a finitely generated $R$-module for every $i \geq 0$. The short exact sequence

$$
0 \rightarrow\left(0: M \mathfrak{a}^{n}\right) \rightarrow M \rightarrow \bar{M} \rightarrow 0
$$

implies that $\operatorname{Ext}_{R}^{i}(R / \mathfrak{a}, M)$ is a finitely generated $R$-module for every $i \geq 0$. 
(ii): Follows from (i).

Lemma 3.10. Let $\mathfrak{a}$ be an ideal of $R$ and $a \in \mathfrak{a}$. If $L$ is an $R$-module such that $L / a L$ and $\left(0:_{L} a\right)$ are $\mathfrak{a}$-Ext-finite, then $L$ is $\mathfrak{a}$-Ext-finite.

Proof. Apply [Me2, Corollary 3.3] to the $R$-homomorphism $f=a \operatorname{Id}_{L}$. We deduce that $L$ is $\mathfrak{a}$-Ext-finite. Note that $\operatorname{Ext}_{R}^{i}(R / \mathfrak{a}, f)=0$ for every $i \geq 0$ as $a \in \mathfrak{a}$.

Lemma 3.11. Let $\mathfrak{a}$ be an ideal of $R$. Suppose that $\operatorname{dim}(R) \leq 2$, and there exists an $a \in \mathfrak{a}$ with $\operatorname{dim}(R / a R) \leq 1$. If $M$ is an $\mathfrak{a}$-Ext-finite $R$-module, then $M / a M$ and $\left(0:_{M}\right.$ a) are $\mathfrak{a}$-Ext-finite.

Proof. Take elements $a_{1}, \ldots, a_{n} \in \mathfrak{a}$ such that $a_{1}=a$ and $\mathfrak{a}=\left(a_{1}, a_{2}, \ldots, a_{n}\right)$. Then by $\mathrm{Me} 2$, Theorem 2.1], $H^{i}\left(a_{1}, \ldots, a_{n} ; M\right)$ is finitely generated for every $i \geq 0$, so it is a-Ext-finite for every $i \geq 0$.

Let $i \geq 0$ and $L:=H^{i}\left(a_{1}, \ldots, a_{n-1} ; M\right)$. Let $\overline{\mathfrak{a}}$ be the image of $\mathfrak{a}$ in $\bar{R}:=R / a R$. By Lemma 3.8. $H^{i}\left(a_{1}, \ldots, a_{n} ; M\right)$ is $\overline{\mathfrak{a}}$-Ext-finite for every $i \geq 0$. In the exact sequence

$$
H^{i}\left(a_{1}, \ldots, a_{n} ; M\right) \rightarrow H^{i}\left(a_{1}, \ldots, a_{n-1} ; M\right) \stackrel{a_{n}}{\longrightarrow} H^{i}\left(a_{1}, \ldots, a_{n-1} ; M\right) \rightarrow H^{i+1}\left(a_{1}, \ldots, a_{n} ; M\right),
$$

the outer terms are $\overline{\mathfrak{a}}$-Ext-finite. By Lemma 3.9 (ii), $L / a_{n} L$ and $\left(0:_{L} a_{n}\right)$ are $\overline{\mathfrak{a}}$-Ext-finite. Hence, Lemma 3.8 implies that $L / a_{n} L$ and $\left(0::_{L} a_{n}\right)$ are a-Ext-finite. By Lemma 3.10, $L$ is $\mathfrak{a}$-Ext-finite.

Continuing in this fashion, we infer that $H^{i}\left(a_{1} ; M\right)$ is a-Ext-finite for every $i \geq 0$, and so $M / a M$ and $\left(0:_{M} a\right)$ are $\mathfrak{a}$-Ext-finite.

Remark 3.12. Let $\mathfrak{a}$ be a proper ideal of $R$ and $f: M \rightarrow N$ an $R$-homomorphism between a-Ext-finite modules. We show that in case we would like to prove that ker $f$ and coker $f$ are $\mathfrak{a}$-Ext-finite, we may additionally assume that $\mathfrak{a}$ contains an $R$-regular element. Indeed, there is an integer $n \geq 1$ such that $\Gamma_{\mathfrak{a}}(R)=\left(0:_{R} \mathfrak{a}^{n}\right)$. Let $\bar{R}=R /\left(0:_{R} \mathfrak{a}^{n}\right)$ and $\overline{\mathfrak{a}}=\mathfrak{a} \bar{R}$. It is clear that $\operatorname{depth}_{\bar{R}}(\overline{\mathfrak{a}}, \bar{R})>0$. Consider the following commutative diagram with exact rows:

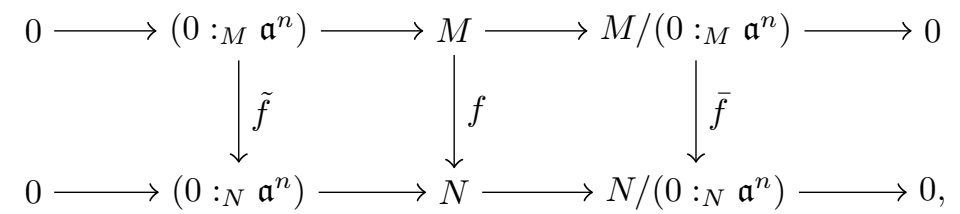

where $\tilde{f}$ and $\bar{f}$ are induced by $f$ in the obvious way. Since the $R$-modules $\left(0::_{M} \mathfrak{a}\right)$ and $\left(0:_{N} \mathfrak{a}\right)$ are finitely generated, it can be seen that the $R$-modules $\left(0:_{M} \mathfrak{a}^{n}\right)$ and $\left(0:_{M} \mathfrak{a}^{n}\right)$ are also finitely generated. So, from the rows of the above commutative diagram, one deduces that the $R$-modues $M /\left(0::_{M} \mathfrak{a}^{n}\right)$ and $N /\left(0:_{N} \mathfrak{a}^{n}\right)$ are $\mathfrak{a}$-Ext-finite.

Applying the Snake Lemma to the above diagram, we get the exact sequence

$$
0 \rightarrow \operatorname{ker} \tilde{f} \rightarrow \operatorname{ker} f \rightarrow \operatorname{ker} \bar{f} \rightarrow \operatorname{coker} \tilde{f} \rightarrow \operatorname{coker} f \rightarrow \operatorname{coker} \bar{f} \rightarrow 0 .
$$

Now, ker $\tilde{f}$ and coker $\tilde{f}$ are finitely generated $R$-modules. Hence, ker $f$ and coker $f$ are a-Extfinite if and only if ker $\bar{f}$ and coker $\bar{f}$ are a-Ext-finite. But, Lemma 3.8 yields that ker $\bar{f}$ and coker $\bar{f}$ are $\mathfrak{a}$-Ext-finite if and only if they are $\overline{\mathfrak{a}}$-Ext-finite.

Finally, we are ready to prove the main result of this section. 
Theorem 3.13. Let $\mathcal{Z}$ be a stable under specialization subset of $\operatorname{Spec}(R)$. Assume that either

(i) $R$ is semilocal with $\operatorname{cd}(\mathcal{Z}, R) \leq 1$, or

(ii) $\operatorname{dim}(\mathcal{Z}) \leq 1$, or

(iii) $\operatorname{dim}(R) \leq 2$.

Then $\mathcal{M}(R, \mathcal{Z})_{\text {cof }}$ is an abelian subcategory of $\mathcal{M}(R)$.

Proof. (i): Let $M$ be an $R$-module, and $N$ a finitely generated $R$-module such that $\operatorname{Supp}_{R}(M) \subseteq \operatorname{Supp}_{R}(N)$. We claim that $\operatorname{cd}(\mathcal{Z}, M) \leq \operatorname{cd}(\mathcal{Z}, N)$. Since $H_{\mathcal{Z}}^{i}(-)$ commutes with direct limits and $M$ can be written as a direct limit of its finitely generated submodules, we may assume that $M$ is finitely generated. Now, the proof is a straightforward adaptation of the argument given in [DNT, Theorem 2.2]. In particular, $\operatorname{cd}(\mathcal{Z}, L) \leq \operatorname{cd}(\mathcal{Z}, R) \leq 1$ for every $R$-module $L$. Thus, the assertion is immediate by Corollary 3.5 and Lemma 3.7 (ii).

(ii): Since $\operatorname{dim}(\mathcal{Z}) \leq 1$, it turns out that $\operatorname{dim}_{R}(M) \leq 1$ for every $\mathcal{Z}$-cofinite $R$-module $M$. Hence, the claim follows from Lemma 3.6 and Lemma 3.7 (i).

(iii): Let $f: M \rightarrow N$ be an $R$-homomorphism between $\mathcal{Z}$-cofinite $R$-modules. We need to show that both ker $f$ and coker $f$ are $\mathfrak{a}$-Ext-finite for every $\mathfrak{a} \in F(\mathcal{Z})$. Fix $\mathfrak{a} \in F(\mathcal{Z})$. In light of Remark 3.12, we may assume that there is an $R$-regular element $a \in \mathfrak{a}$. Besides, in view of the short exact sequences

$$
0 \longrightarrow \operatorname{ker} f \longrightarrow M \longrightarrow \operatorname{im} f \longrightarrow 0
$$

and

$$
0 \longrightarrow \operatorname{im} f \longrightarrow N \longrightarrow \operatorname{coker} f \longrightarrow 0,
$$

it is sufficient to show that $\operatorname{im} f$ is a-Ext-finite. The $R$-modules $M / a M$ and $\left(0::_{N} a\right)$ are $\mathfrak{a}$-Ext-finite by Lemma 3.11. Hence Lemma 3.8 implies that $M / a M$ and $\left(0:_{N} a\right)$ are $\mathfrak{a} / a R$-Extfinite over the $\operatorname{ring} \bar{R}:=R / a R$ which has dimension at most one. We note that $\operatorname{im} f / a \operatorname{im} f$ is a homomorphic image of $M / a M$, and $(0: \operatorname{im} f a)$ is a submodule of $\left(0:_{N} a\right)$. Therefore, $\operatorname{im} f / a \operatorname{im} f$ and $(0: \operatorname{im} f a)$ are $\mathfrak{a} / a R$-Ext-finite by Lemma 3.9 (ii). Hence, they are $\mathfrak{a}$-Ext-finite by Lemma 3.8. Now, Lemma 3.10 implies that $\operatorname{im} f$ is a-Ext-finite.

In Theorem 3.13 (i), the assumption that $R$ is semilocal is somehow not desirable. Accordingly, we pose the following question.

Question 3.14. Let $\mathcal{Z}$ be a stable under specialization subset of $\operatorname{Spec}(R)$ such that $\operatorname{cd}(\mathcal{Z}, R) \leq 1$. Is $\mathcal{M}(R, \mathcal{Z})_{\text {cof }}$ an abelian subcategory of $\mathcal{M}(R)$ ?

\section{Proof of Theorem $\mathbf{1 . 6}$}

In this section, we intend to prove Theorem 1.6, see Theorem 4.7. To this end, Lemma 4.6 is our main tool. In order to apply it in the situation of Theorem 4.7, one has to use Theorem 3.13, and Lemmas 4.3 and 3.7. For proving Lemma 4.3, we need Lemmas 4.1 and 4.2 .

Given a stable under specialization subset $\mathcal{Z}$ of $\operatorname{Spec}(R)$ and a finitely generated $R$-module $M$, we remind that

$$
\operatorname{depth}_{R}(\mathcal{Z}, M):=\inf \left\{\operatorname{depth}_{R}(\mathfrak{a}, M) \mid \mathfrak{a} \in F(\mathcal{Z})\right\}
$$

Lemma 4.1. Let $\mathcal{Z}$ be a stable under specialization subset of $\operatorname{Spec}(R)$, and $M$ a finitely generated $R$-module. Then $\operatorname{Supp}_{R}(M) \cap \mathcal{Z}=\bigcup_{i=0}^{\infty} \operatorname{Supp}_{R}\left(H_{\mathcal{Z}}^{i}(M)\right)$. 
Proof. Let $\mathfrak{p} \in \operatorname{Supp}_{R}(M) \cap \mathcal{Z}$. It is straightforward to see that the set

$$
\mathcal{Z}_{\mathfrak{p}}:=\left\{\mathfrak{q} R_{\mathfrak{p}} \mid \mathfrak{q} \in \mathcal{Z} \text { and } \mathfrak{q} \subseteq \mathfrak{p}\right\}
$$

is a stable under specialization $\operatorname{subset}$ of $\operatorname{Spec}\left(R_{\mathfrak{p}}\right)$. It is clear that $\mathfrak{p} R_{\mathfrak{p}} \in \mathcal{Z}_{\mathfrak{p}} \cap \operatorname{Supp}_{R_{\mathfrak{p}}}\left(M_{\mathfrak{p}}\right)$, so $\operatorname{depth}_{R_{\mathfrak{p}}}\left(\mathfrak{p} R_{\mathfrak{p}}, M_{\mathfrak{p}}\right)<\infty$, and thus $s:=\operatorname{depth}_{R_{\mathfrak{p}}}\left(\mathcal{Z}_{\mathfrak{p}}, M_{\mathfrak{p}}\right)<\infty$. However by [Bi, Proposition $5.5]$

$$
\operatorname{depth}_{R_{\mathfrak{p}}}\left(\mathcal{Z}_{\mathfrak{p}}, M_{\mathfrak{p}}\right)=\inf \left\{i \in \mathbb{Z} \mid H_{\mathcal{Z}_{\mathfrak{p}}}^{i}\left(M_{\mathfrak{p}}\right) \neq 0\right\}
$$

so $H_{\mathcal{Z}_{\mathfrak{p}}}^{s}\left(M_{\mathfrak{p}}\right) \neq 0$. One may check that $H_{\mathcal{Z}}^{s}(M)_{\mathfrak{p}} \cong H_{\mathcal{Z}_{\mathfrak{p}}}^{s}\left(M_{\mathfrak{p}}\right)$, and so $\mathfrak{p} \in$ $\bigcup_{i=0}^{\infty} \operatorname{Supp}_{R}\left(H_{\mathcal{Z}}^{i}(M)\right)$. The reverse inclusion is immediate.

Lemma 4.2. Let $\mathcal{Z}$ be a stable under specialization subset of $\operatorname{Spec}(R)$, and $M$ a finitely generated $R$-module. Then $\operatorname{dim}\left(\operatorname{Supp}_{R}\left(H_{\mathcal{Z}}^{i}(M)\right)\right) \leq \operatorname{dim}_{R}(M)-1$ for every $i \geq 1$.

Proof. Clearly, we may assume that $\operatorname{dim}_{R}(M)<\infty$. Since

$$
H_{\mathcal{Z}}^{i}(M) \cong \underset{\mathfrak{a} \in F(\mathcal{Z})}{\lim _{\mathfrak{a}}} H_{\mathfrak{a}}^{i}(M)
$$

for every $i \geq 0$, it suffices to show that $\operatorname{dim}\left(\operatorname{Supp}_{R}\left(H_{\mathfrak{a}}^{i}(M)\right)\right) \leq \operatorname{dim}_{R}(M)-1$ for every $\mathfrak{a} \in F(\mathcal{Z})$ and every $i \geq 1$. As $H_{\mathfrak{a}}^{i}(M) \cong H_{\mathfrak{a}}^{i}\left(M / \Gamma_{\mathfrak{a}}(M)\right)$ for every $i \geq 1$, we may assume that $\Gamma_{\mathfrak{a}}(M)=0$. Consequently, we conclude that $\mathfrak{a}$ contains a nonzerodivisor $r$ on $M$. As $\operatorname{Supp}_{R}\left(H_{\mathfrak{a}}^{i}(M)\right) \subseteq \operatorname{Supp}_{R}(M / \mathfrak{a} M)$, we have

$$
\begin{aligned}
\operatorname{dim}\left(\operatorname{Supp}_{R}\left(H_{\mathfrak{a}}^{i}(M)\right)\right) & \leq \operatorname{dim}_{R}(M / \mathfrak{a} M) \\
& \leq \operatorname{dim}_{R}(M / r M) \\
& \leq \operatorname{dim}_{R}(M)-1
\end{aligned}
$$

for every $i \geq 1$.

Lemma 4.3. Let $\mathcal{Z}$ be a stable under specialization subset of $\operatorname{Spec}(R)$, and $M$ a finitely generated $R$-module. Assume that either

(i) $\operatorname{cd}(\mathcal{Z}, R) \leq 1$, or

(ii) $\operatorname{dim}(\mathcal{Z}) \leq 1$, or

(iii) $\operatorname{dim}\left(\operatorname{Supp}_{R}\left(H_{\mathcal{Z}}^{i}(M)\right)\right) \leq 1$ for every $i \geq 0$, or

(iv) $\operatorname{dim}_{R}(M) \leq 2$.

Then $H_{\mathcal{Z}}^{i}(M)$ is $\mathcal{Z}$-cofinite for every $i \geq 0$.

Proof. (i): See [MS, Proposition 2.7].

(ii): Clearly, $\operatorname{Supp}_{R}\left(H_{\mathcal{Z}}^{i}(M)\right) \subseteq \mathcal{Z}$ for every $i \geq 0$. Thus, it remains to show that $\operatorname{Ext}_{R}^{j}\left(R / \mathfrak{a}, H_{\mathcal{Z}}^{i}(M)\right)$ is finitely generated for every $\mathfrak{a} \in F(\mathcal{Z})$ and every $i, j \geq 0$.

Fix $\mathfrak{b} \in F(\mathcal{Z})$. By induction on $i$, for a given $R$-module $N$ with $\operatorname{Ext}_{R}^{j}(R / \mathfrak{b}, N)$ finitely generated for every $j \geq 0$, we show that $\operatorname{Ext}_{R}^{j}\left(R / \mathfrak{b}, H_{\mathcal{Z}}^{i}(N)\right)$ is finitely generated for every $j \geq 0$. The short exact sequence

$$
0 \rightarrow \Gamma_{\mathcal{Z}}(N) \rightarrow N \rightarrow M / \Gamma_{\mathcal{Z}}(N) \rightarrow 0,
$$


yields the exact sequence

$$
\begin{gathered}
0 \rightarrow \operatorname{Hom}_{R}\left(R / \mathfrak{b}, \Gamma_{\mathcal{Z}}(N)\right) \rightarrow \operatorname{Hom}_{R}(R / \mathfrak{b}, N) \rightarrow \operatorname{Hom}_{R}\left(R / \mathfrak{b}, N / \Gamma_{\mathcal{Z}}(N)\right) \rightarrow \\
\operatorname{Ext}_{R}^{1}\left(R / \mathfrak{b}, \Gamma_{\mathcal{Z}}(N)\right) \rightarrow \operatorname{Ext}_{R}^{1}(R / \mathfrak{b}, N) .
\end{gathered}
$$

It can be seen by inspection that $\operatorname{Hom}_{R}\left(R / \mathfrak{b}, N / \Gamma_{\mathcal{Z}}(N)\right)=0$. Hence the above exact sequence shows that the $R$-modules $\operatorname{Hom}_{R}\left(R / \mathfrak{b}, \Gamma_{\mathcal{Z}}(N)\right)$ and $\operatorname{Ext}_{R}^{1}\left(R / \mathfrak{b}, \Gamma_{\mathcal{Z}}(N)\right)$ are finitely generated. Therefore, by Lemma 3.6 the case $i=0$ holds true.

Now, suppose that $i \geq 1$ and make the obvious induction hypothesis. From the exact sequence

$$
\operatorname{Ext}_{R}^{j}(R / \mathfrak{b}, N) \rightarrow \operatorname{Ext}_{R}^{j}\left(R / \mathfrak{b}, N / \Gamma_{\mathcal{Z}}(N)\right) \rightarrow \operatorname{Ext}_{R}^{j+1}\left(R / \mathfrak{b}, \Gamma_{\mathcal{Z}}(N)\right),
$$

using the base case $i=0$, we deduce that $\operatorname{Ext}_{R}^{j}\left(R / \mathfrak{b}, N / \Gamma_{\mathcal{Z}}(N)\right)$ is finitely generated for every $j \geq 0$. Since $H_{\mathcal{Z}}^{i}(N) \cong H_{\mathcal{Z}}^{i}\left(N / \Gamma_{\mathcal{Z}}(N)\right)$ for every $i \geq 1$, we may assume that $\Gamma_{\mathcal{Z}}(N)=0$. Let $E:=E_{R}(N)$ and $L:=E / N$. We have $\Gamma_{\mathcal{Z}}(E) \cong E_{R}\left(\Gamma_{\mathcal{Z}}(N)\right)=0$, and $\operatorname{Hom}_{R}(R / \mathfrak{b}, E)=0$. Then from the short exact sequence

$$
0 \rightarrow N \rightarrow E \rightarrow L \rightarrow 0
$$

we conclude that $H_{\mathcal{Z}}^{k}(N) \cong H_{\mathcal{Z}}^{k-1}(L)$ and $\operatorname{Ext}_{R}^{k}(R / \mathfrak{b}, N) \cong \operatorname{Ext}_{R}^{k-1}(R / \mathfrak{b}, L)$ for every $k \geq 1$. Hence the assumption is satisfied by $L$, and thus $\operatorname{Ext}_{R}^{j}\left(R / \mathfrak{b}, H_{\mathcal{Z}}^{i-1}(L)\right)$ is finitely generated for every $j \geq 0$.

(iii): By the assumption and Lemma 4.1, we have $\operatorname{dim}\left(\operatorname{Supp}_{R}(M) \cap \mathcal{Z}\right) \leq 1$. Set

$$
\widetilde{\mathcal{Z}}:=\left\{\frac{\mathfrak{p}}{\operatorname{ann}_{R}(M)} \mid \mathfrak{p} \in \operatorname{Supp}_{R}(M) \cap \mathcal{Z}\right\},
$$

and $S:=R / \operatorname{ann}_{R}(M)$. Then, it is straightforward to see that $\widetilde{\mathcal{Z}}$ is a stable under specialization subset of $\operatorname{Spec}(S)$ with $\operatorname{dim}(\widetilde{\mathcal{Z}}) \leq 1$, and $F(\widetilde{\mathcal{Z}})=\{\mathfrak{a} S \mid \mathfrak{a} \in F(\mathcal{Z})\}$. In addition, we have

$$
\begin{aligned}
& H_{\mathcal{Z}}^{i}(M) \cong \underset{\mathfrak{a} \in F(\mathcal{Z})}{\lim _{\mathfrak{a}}} H_{\mathfrak{a}}^{i}(M) \\
& \cong \lim _{\mathfrak{a} \in F(\mathcal{Z})} H_{\mathfrak{a} S}^{i}(M) \\
& \cong \lim _{\mathfrak{b} \in F(\tilde{\mathcal{Z}})} H_{\mathfrak{b}}^{i}(M) \\
& \cong H_{\widetilde{\mathcal{Z}}}^{i}(M) \text {. }
\end{aligned}
$$

for every $i \geq 0$. Hence by part (ii), $N:=H_{\mathcal{Z}}^{i}(M) \cong H_{\widetilde{\mathcal{Z}}}^{i}(M)$ is a $\widetilde{\mathcal{Z}}$-cofinite $S$-module for every $i \geq 0$. Now, Lemma 3.8 implies that $H_{\mathcal{Z}}^{i}(M)$ is $\mathcal{Z}$-cofinite for every $i \geq 0$.

(iv): Clearly, $\Gamma_{\mathcal{Z}}(M)$ is $\mathcal{Z}$-cofinite. So by replacing $M$ with $M / \Gamma_{\mathcal{Z}}(M)$, we can assume that $H_{\mathcal{Z}}^{0}(M)=0$. Now, Lemma 4.2 implies that $\operatorname{dim}\left(\operatorname{Supp}_{R}\left(H_{\mathcal{Z}}^{i}(M)\right)\right) \leq 1$ for all $i \geq 0$, thereby part (iii) completes the argument.

In the rest of this section, we apply the technique of way-out functors to prove the main result of this section.

Definition 4.4. Let $R$ and $S$ be two rings, and $\mathcal{F}: \mathcal{D}(R) \rightarrow \mathcal{D}(S)$ a covariant functor. We say that $\mathcal{F}$ is way-out left if for every $n \in \mathbb{Z}$, there is an $m \in \mathbb{Z}$, such that for any $R$-complex $X$ with $\sup X \leq m$, we have $\sup \mathcal{F}(X) \leq n$. 
The Way-out Lemma appears in [Ha2, Ch. I, Proposition 7.3]. However, we need a refined version which is tailored to our needs. Let $R$ and $S$ be two rings. Roughly speaking, a triangulated functor is a functor $\mathcal{F}: \mathcal{D}(R) \rightarrow \mathcal{D}(S)$ that preserves shift and distinguished triangles; See CFH, Definition A.7].

Lemma 4.5. Let $R$ and $S$ be two rings, and $\mathcal{F}: \mathcal{D}(R) \rightarrow \mathcal{D}(S)$ a triangulated covariant functor. Let $\mathcal{A}$ be an additive subcategory of $\mathcal{M}(R)$, and $\mathcal{B}$ an abelian subcategory of $\mathcal{M}(S)$ which is closed under extensions. Suppose that $H_{i}(\mathcal{F}(M)) \in \mathcal{B}$ for every $M \in \mathcal{A}$ and every $i \in \mathbb{Z}$. If $\mathcal{F}$ is way-out left and $X \in \mathcal{D}_{\sqsubset}(R)$ with $H_{i}(X) \in \mathcal{A}$ for every $i \in \mathbb{Z}$, then $H_{i}(\mathcal{F}(X)) \in \mathcal{B}$ for every $i \in \mathbb{Z}$.

Proof. See [DFT, Lemma 3.2].

The next result provides us with a suitable transition device from modules to complexes when dealing with cofiniteness.

Lemma 4.6. Let $\mathcal{Z}$ a stable under specialization subset of $\operatorname{Spec}(R)$. Then the functor $\mathbf{R} \Gamma_{\mathcal{Z}}(-)$ : $\mathcal{D}(R) \rightarrow \mathcal{D}(R)$ is triangulated and way-out left. As a consequence, if $H_{\mathcal{Z}}^{i}(M)$ is $\mathcal{Z}$-cofinite for every finitely generated $R$-module $M$ and every $i \geq 0$, and $\mathcal{M}(R, \mathcal{Z})_{\text {cof }}$ is an abelian category, then $H_{\mathcal{Z}}^{i}(X)$ is $\mathcal{Z}$-cofinite for every $X \in \mathcal{D}_{\sqsubset}^{f}(R)$ and every $i \in \mathbb{Z}$.

Proof. It is folklore that if an endofunctor on $\mathcal{D}(R)$ extends from an endofunctor on $\mathcal{M}(R)$, then it commutes with mapping cones. Hence, it can be easily verified that the functor $\mathbf{R} \Gamma_{\mathcal{Z}}(-)$ : $\mathcal{D}(R) \rightarrow \mathcal{D}(R)$ is triangulated. Moreover, if $\sup X \leq n$, then there is a semi-injective resolution $X \stackrel{\simeq}{\rightarrow} I$ of $X$ such that $I_{i}=0$ for every $i \geq n$ due to [CFH, Theorem 5.3.26]. Applying the functor $\Gamma_{\mathcal{Z}}(-)$ to $I$ and taking homology, we see that $\sup \mathbf{R} \Gamma_{\mathcal{Z}}(X) \leq n$. It follows that the functor $\mathbf{R} \Gamma_{\mathcal{Z}}(-)$ is way-out left. Now, let $\mathcal{A}$ be the subcategory of finitely generated $R$-modules, and let $\mathcal{B}:=\mathcal{M}(R, \mathcal{Z})_{\text {cof }}$. It can be easily seen that $\mathcal{B}$ is closed under extensions. It now follows from Lemma 4.5 that $H_{\mathcal{Z}}^{i}(X)=H_{-i}\left(\mathbf{R} \Gamma_{\mathcal{Z}}(X)\right) \in \mathcal{B}$ for every $X \in \mathcal{D}_{\sqsubset}^{f}(R)$ and every $i \in \mathbb{Z}$.

Theorem 4.7. Let $\mathcal{Z}$ a stable under specialization subset of $\operatorname{Spec}(R)$, and $X \in \mathcal{D}_{\sqsubset}^{f}(R)$. Assume that either

(i) $R$ is semilocal with $\operatorname{cd}(\mathcal{Z}, R) \leq 1$, or

(ii) $\operatorname{dim}(\mathcal{Z}) \leq 1$, or

(iii) $\operatorname{dim}\left(\operatorname{Supp}_{R}(X)\right) \leq 2$.

Then $H_{\mathcal{Z}}^{i}(X)$ is $\mathcal{Z}$-cofinite for every $i \in \mathbb{Z}$.

Proof. (i) and (ii): In view of Theorem 3.13 and Lemma 4.3, one can apply Lemma 4.6 to conclude the claim.

(iii): Let

$$
\mathcal{A}:=\left\{M \in \mathcal{M}(R) \mid M \text { is finitely generated and } \operatorname{dim}_{R}(M) \leq 2\right\},
$$

and

$$
\mathcal{B}:=\left\{M \in \mathcal{M}(R) \mid M \text { is } \mathcal{Z} \text {-cofinite and } \operatorname{dim}_{R}(M) \leq 1\right\} .
$$

By Lemma 3.6 and Lemma 3.7 (i), $\mathcal{B}$ is an abelian subcategory of $\mathcal{M}(R)$. In addition, it is closed under extensions. Now, by Lemmas 4.2 and 4.3 (iv), we have $H_{\mathcal{Z}}^{i}(M) \in \mathcal{B}$ for any $M \in \mathcal{A}$. Considering the triangulated way-out left functor $\mathbf{R} \Gamma_{\mathcal{Z}}(-)$, Lemma 4.5 yields that $H_{\mathcal{Z}}^{i}(X)=H_{-i}\left(\mathbf{R} \Gamma_{\mathcal{Z}}(X)\right) \in \mathcal{B}$ for every $X \in \mathcal{D}_{\sqsubset}(R)$ with $H_{i}(X) \in \mathcal{A}$ and for every $i \in \mathbb{Z}$. 
Remark 4.8. Given a stable under specialization subset $\mathcal{Z}$ of $\operatorname{Spec}(R)$, the local cohomology module $H_{\mathcal{Z}}^{i}(X)$ of an $R$-complex $X$ with support in $\mathcal{Z}$, is an all-in-one generalization of the previously known generalized local cohomology modules as outlined in the following discussion.

(i) Let $\mathfrak{a}$ be an ideal of $R$, and $M$ and $N$ two $R$-modules. The generalized local cohomology module $H_{\mathfrak{a}}^{i}(M, N)$ is defined as

$$
H_{\mathfrak{a}}^{i}(M, N):=\underset{n}{\lim } \operatorname{Ext}_{R}^{i}\left(M / \mathfrak{a}^{n} M, N\right)
$$

for every $i \geq 0$; see $\mathrm{Gr}$ and $[\mathrm{He}$. It is shown in [Ya] that if $M$ is finitely generated, then we have $H_{\mathfrak{a}}^{i}(M, N)=H_{\mathcal{Z}}^{i}(X)$ for every $i \geq 0$, where $\mathcal{Z}=V(\mathfrak{a})$ and $X=\mathbf{R} \operatorname{Hom}_{R}(M, N)$.

(ii) Let $\mathfrak{a}$ and $\mathfrak{b}$ be two ideals of $R$, and $M$ an $R$-module. Let

$$
W(\mathfrak{a}, \mathfrak{b}):=\left\{\mathfrak{p} \in \operatorname{Spec}(R) \mid \mathfrak{a}^{n} \subseteq \mathfrak{p}+\mathfrak{b} \text { for some integer } n \geq 1\right\} .
$$

Define a functor $\Gamma_{\mathfrak{a}, \mathfrak{b}}(-)$ on $\mathcal{M}(R)$ by setting

$$
\Gamma_{\mathfrak{a}, \mathfrak{b}}(M):=\left\{x \in M \mid \operatorname{Supp}_{R}(R x) \subseteq W(\mathfrak{a}, \mathfrak{b})\right\},
$$

for an $R$-module $M$, and $\Gamma_{\mathfrak{a}, \mathfrak{b}}(f):=\left.f\right|_{\Gamma_{\mathfrak{a}, \mathfrak{b}}(M)}$ for an $R$-homomorphism $f: M \rightarrow N$. The generalized local cohomology module $H_{\mathfrak{a}, \mathfrak{b}}^{i}(M)$ is defined in TYY] to be $H_{\mathfrak{a}, \mathfrak{b}}^{i}(M):=$ $R^{i} \Gamma_{\mathfrak{a}, \mathfrak{b}}(M)$ for every $i \geq 0$. It is clear that $H_{\mathfrak{a}, \mathfrak{b}}^{i}(M)=H_{\mathcal{Z}}^{i}(X)$ for every $i \geq 0$, where $\mathcal{Z}=W(\mathfrak{a}, \mathfrak{b})$ and $X=M$.

(iii) Let $\Phi$ be a directed poset. By a system of ideals $\varphi$, we mean a family $\varphi=\left\{\mathfrak{a}_{\alpha}\right\}_{\alpha \in \Phi}$ of ideals of $R$, such that $\mathfrak{a}_{\alpha} \subseteq \mathfrak{a}_{\beta}$ whenever $\alpha \geq \beta$, and for any $\alpha, \beta \in \Phi$, there is a $\gamma \in \Phi$ with $\mathfrak{a}_{\gamma} \subseteq \mathfrak{a}_{\alpha} \mathfrak{a}_{\beta}$. Given a system of ideals $\varphi$, define a functor $\Gamma_{\varphi}(-)$ on $\mathcal{M}(R)$ by setting

$$
\Gamma_{\varphi}(M):=\{x \in M \mid \mathfrak{a} x=0 \text { for some } \mathfrak{a} \in \varphi\},
$$

for an $R$-module $M$, and $\Gamma_{\varphi}(f):=\left.f\right|_{\Gamma_{\varphi}(M)}$ for an $R$-homomorphism $f: M \rightarrow N$. Then the generalized local cohomology module $H_{\varphi}^{i}(M)$ is defined in [BS, Notation 2.2.2] to be $H_{\varphi}^{i}(M):=R^{i} \Gamma_{\varphi}(M)$ for every $i \geq 0$. It is easy to see that $H_{\varphi}^{i}(M)=H_{\mathcal{Z}}^{i}(X)$ for every $i \geq 0$, where $\mathcal{Z}=\bigcup_{\mathfrak{a} \in \varphi} V(\mathfrak{a})$ and $X=M$.

(iv) Yoshino and Yoshizawa $[\mathrm{YY}$, Theorem 2.10] have shown that for any abstract local cohomology functor $\delta: \mathcal{D}_{\sqsubset}(R) \rightarrow \mathcal{D}_{\sqsubset}(R)$, there is a stable under specialization subset $\mathcal{Z}$ of $\operatorname{Spec}(R)$ such that $\delta \cong \mathbf{R} \Gamma_{\mathcal{Z}}(-)$.

Accordingly, our Theorems 1.5 and 1.6 generalize the following results:

(a) [HV, Proposition 3.6, Corollaries 3.9, 3.10, 3.11, and 3.12].

(b) [DH, Theorem 2.5].

(c) [DS, Theorem 1.3].

(d) [TGV, Theorems 1.1 and 1.3].

(e) [Ha1, Propositions 6.1 and 7.6, and Corollaries 6.3 and 7.7].

(f) Ka1, Theorem 2.1].

(g) [Ka2, Theorem 1].

\section{References}

[AB] M. Aghapournahr and K. Bahmanpour, Cofiniteness of general local cohomology modules for small dimensions, Bull. Korean Math. Soc., 53(5), (2016), 1341-1352. 
[AF] L. Avramov and H-B. Foxby, Homological dimensions of unbounded complexes, J. Pure Appl. Algebra, 71(2-3), (1991), 129-155.

[BNS] K. Bahmanpour, R. Naghipour and M. Sedghi, On the category of cofinite modules which is abelian, Proc. Amer. Math. Soc., 142(4), (2014), 1101-1107.

[BN] K. Bahmanpour and R. Naghipour, Cofiniteness of local cohomology modules for ideals of small dimension, J. Algebra, 321(7), (2009), 1997-2011.

[BS] M. Brodmann and R.Y. Sharp, Local cohomology: An algebraic introduction with geometric applications, Cambridge Studies in Advanced Mathematics, 136, Cambridge University Press, Cambridge, Second Edition (2013).

[Bi] M.H. Bijan-Zadeh, A common generalization of local cohomology theories, Glasgow Math. J., 21(2), (1980), 173-181.

[CFH] L. W. Christensen, H.-B. Foxby, and H. Holm, Derived category methods in commutative algebra, draft November 13, 2012.

[CW] F.C. Cheng and M.Y. Wang, Homological dimension of G-Matlis dual modules over semilocal rings, Comm. Algebra, 21(4), (1993), 1215-1220.

[DM] D. Delfino and T. Marley, Cofinite modules and local cohomology, J. Pure Appl. Algebra, 121(1), (1997), 45-52.

[DFT] K. Divaani-Aazar, H. Faridian, and M. Tousi, A New Outlook on Cofiniteness, arXiv:1701.07716 [math.AC].

[DH] K. Divaani-Aazar and A. Hajikarimi, Cofiniteness of generalized local cohomology modules for onedimensional ideals, Canad. Math. Bull., 55(1), (2012), 81-87.

[DNT] K. Divaani-Aazar, R. Naghipour and M. Tousi, Cohomological dimension of certain algebraic varieties, Proc. Amer. Math. Soc., 130(12), (2002), 3537-3544.

[DS] K. Divaani-Aazar and R. Sazeedeh, Cofiniteness of generalized local cohomology modules, Colloq. Math., 99(2), (2004), 283-290.

[Gr] A. Grothendieck, Cohomologie locale des faisceaux cohèrents et thèorèmes de Lefschetz locaux et globaux, (SGA 2), North-Holland, Amsterdam, 1968.

[Ha1] R. Hartshorne, Affine duality and cofiniteness, Invent. Math., 9, (1969/1970), 145-164.

[Ha2] R. Hartshorne, Residues and duality, Lecture Notes in Mathematics, 20, (1966).

[HD] M. Hatamkhani and K. Divaani-Aazar, The derived category analogue of the Hartshorne-Lichtenbaum vanishing theorem, Tokyo. J. Math., 36(1), (2013), 195-205.

[He] J. Herzog, Komplexe, auflosungen und dualitat in der lokalen algebra, Habilitationsschrift, Universitat Regensburg, (1970).

[HV] S. H. Hasanzadeh and A. Vahidi, On vanishing and cofiniteness of generalized local cohomology modules, Comm. Algebra, 37(7), (2009), 2290-2299.

[HK] G. Huneke and J. Koh, Cofiniteness and vanishing of local cohomology modules, Math. Proc. Camb. Phil. Soc., 110(3), (1991), 421-429.

[HS] G. Huneke and R.Y. Sharp, Bass numbers of local cohomology modules, Trans. Amer. Math. Soc., 339(2), (1993), 765-779.

[Ka1] K. Kawasaki, On the category of cofinite modules for principal ideals, Nihonkai Math. J., 22(2), (2011), $67-71$.

[Ka2] K. Kawasaki, Cofiniteness of local cohomology modules for principal ideals, Bull. London Math. Soc., 30(3), (1998), 241-246.

[Li] J. Lipman, Lectures on local cohomology and duality, Local cohomology and its applications, Lecture notes in pure and applied mathematics, 226, (2012), Marcel Dekker, Inc.

[Ly] G. Lyubeznik, Finiteness properties of local cohomology modules (an application of D-modules to commutative algebra), Invent. Math., 113(1), (1993), 41-55.

[MS] A. Mafi and H. Saremi, On the cofiniteness properties of certain general local cohomology modules, Acta Sci. Math. (Szeged), 74,(3-4), (2008), 501-507.

[Me1] L. Melkersson, Cofiniteness with respect to ideals of dimension one, J. Algebra, 372, (2012), 459-462.

[Me2] L. Melkersson, Modules cofinite with respect to an ideal, J. Algebra, 285(2), (2005), 649-668. 
[Me3] L. Melkersson, On asymptotic stability for sets of prime ideals connected with the powers of an ideal, Math. Proc. Cambridge Philos. Soc., 107(2), (1990), 267-271.

[Ro] J.J. Rotman, An introduction to homological algebra, Universitext. Springer, New York, second edition, 2009.

[Sp] N. Spaltenstein, Resolutions of unbounded complexes, Compositio Math., 65(2), (1988), $121-154$.

[TGV] N. Tu Cuong, S. Goto and N. Van Hoang, On the cofiniteness of generalized local cohomology modules, Kyoto Journal of Mathematics, 55(1), (2015), 169-185.

[TYY] R. Takahashi, Y. Yoshino and T. Yoshizawa, Local cohomology based on a nonclosed support defined by a pair of ideals, J. Pure Appl. Algebra, 213(4), (2009), 582-600.

[WW] S. Sather-Wagstaff and R. Wicklein, Support and adic finiteness for complexes, Comm. Algebra, 45(6), (2017), 2569-2592.

[Ya] S. Yassemi, Generalized section functors, J. Pure Appl. Algebra, 95(1), (1994), 103-119.

[YY] Y. Yoshino and T. Yoshizawa, Abstract local cohomology functors, Math. J. Okayama Univ., 53, (2011), 129-154.

K. Divanini-Aazar, Department of Mathematics, Alzahra University, Vanak, Post Code 19834, Tehran, Iran-And-School of Mathematics, Institute for Research in Fundamental Sciences (IPM), P.O. BOX 19395-5746, TEHRAN, IRAN.

E-mail address: kdivaani@ipm.ir

H. Faridian, Department of Mathematics, Shahid Beheshti University, G.C., Evin, Tehran, Iran, ZIP CODE 1983963113.

E-mail address: h.faridian@yahoo.com

M. Tousi, Department of Mathematics, Shahid Beheshti University, G.C., Evin, Tehran, Iran, P.O. Box 19395-5746.

E-mail address: mtousi@ipm.ir 